\title{
A cross-sectional investigation of the health needs of asylum seekers in a refugee clinic in Germany
}

\author{
Laura F. Goodman', Guy W. Jensen 1 , Joseph M. Galante', Diana L. Farmer ${ }^{1}$ and Stephanie Taché ${ }^{2^{*}}$ (D)
}

\begin{abstract}
Background: Over one million asylum seekers were registered in Germany in 2016, most from Syria and Afghanistan. The Refugee Convention guarantees access to healthcare, however delivery mechanisms remain heterogeneous. There is an urgent need for more data describing the health conditions of asylum seekers to guide best practices for healthcare delivery. In this study, we describe the state of health of asylum seekers presenting to a multi-specialty primary care refugee clinic.

Methods: Demographic and medical diagnosis data were extracted from the electronic medical records of patients seen at the ambulatory refugee clinic in Dresden, Germany between 15 September 2015 and 31 December 2016. Data were de-identified and analyzed using Stata version 14.0.

Results: Two-thousand-seven-hundred and fifty-three individual patients were seen in the clinic. Of these, 2232 (81.1\%) were insured by the state indicating arrival within the last 3 months. The median age was 25, interquartile range 16-34. Only 786 (28.6\%) were female, while 1967 (71.5\%) were male. The most frequent diagnoses were respiratory (17.4\%), followed by miscellaneous symptoms and otherwise not classified ailments (R series, 14.1\%), infection (10.8\%), musculoskeletal or connective tissue (9.3\%), gastrointestinal (6.8\%), injury (5.9\%), and mental or behavioral (5.1\%) categories.

Conclusions: This study illustrates the diverse medical conditions that affect the asylum seeker population. Asylum seekers in our study group did not have a high burden of communicable diseases, however several warranted additional screening and treatment, including for tuberculosis and scabies. Respiratory illnesses were more common amongst newly arrived refugees. Trauma-related mental health disorders comprised half of mental health diagnoses.
\end{abstract}

Keywords: Asylum seeker, Refugee, Public health, Health care, Health systems, Epidemiology

\section{Background}

There were 65.5 million displaced persons worldwide in 2016 [1]. Approximately 1.3 million people applied for asylum in Europe in 2016 [2], most fleeing from Syria, Afghanistan and South Sudan [3]. Germany was the primary destination for asylum seekers in 2016, receiving 722,400 out of the 2.8 million applications worldwide [3].

\footnotetext{
* Correspondence: stephtache1@gmail.com

${ }^{2}$ General Medicine Division, MK3, Technical University Dresden, Carus School of Medicine, Fetscherstrasse 74, 01307 Dresden, Germany

Full list of author information is available at the end of the article
}

Asylum seekers in Germany are excluded from routine health monitoring systems such that the body of knowledge on refugee health status in Germany is limited [4]. However, a small number of studies describe a broad range of diagnoses $[5,6]$. A systematic review of studies on health status and medical care among refugees and asylum seekers in Germany identified three publications not using a disease-based approach for comparison [4]. The remaining available studies focused on specific specialties such as infectious diseases, psychiatry, or special populations such as minors. A recent study of health conditions amongst asylum seekers at a camp in Brussels found that upper airway infections were the most

(c) The Author(s). 2018 Open Access This article is distributed under the terms of the Creative Commons Attribution 4.0 International License (http://creativecommons.org/licenses/by/4.0/), which permits unrestricted use, distribution, and 
common diagnoses, followed by dental caries, and skin infection [5]. A 2014 review of non-communicable diseases (NCDs) among urban refugees in developing countries found the prevalence of NCDs amongst those from the Middle East ranged from 9 to 50\% [7]. Another recent review noted that the prevalence of TB in Syria, the country of origin of most asylum seekers in Germany in 2015, was lower than that in several European Union countries [8]. A small pilot study of disease prevalence among youth asylum seekers in Germany found that over half had infections, nearly half of which were Helicobacter pylori [9].

In response to the influx of asylum seekers in 2015, Germany mounted a nationwide response to secure basic needs including medical care for this population [10] (see Fig. 1). In this study, asylum seeker refers to both those who have received formal asylum (refugees) and those who have applied for refugee status. The state of Saxony received approximately 24,000 asylum-seekers in the second half of 2015 [11]. In response, three ambulatory refugee clinics were created in Saxony. The first was opened in September 2015 in Dresden [12, 13].

Our study describes the medical diagnoses among asylum seekers in one clinic in Dresden. Through this study, we aim to add to the nascent epidemiological data characterizing this population.

\section{Methods}

\section{Materials, methods, and setting}

Implemented as a partnership between the city of Dresden, the state of Saxony, and the regulating union for outpatient care (Kassenärztliche Vereinigung Sachsen or KVS), the refugee clinic functioned as a first medical triage and care point for asylum seekers in Dresden. It provided on-site diagnostic and treatment services in general medicine, pediatrics, and psychiatry.

\section{Data source}

Data for all patients seen in the asylum-seeker clinic in the period 14 September 2015-31 December 2015 were extracted from the electronic medical record (Turbomed) and de-identified by the staff at KVS. Age, gender, and insurance category, were merged with diagnosis(es) with International Classification of Disease-10 (ICD-10) codes and clinic visit code. Unlinked data on country of origin of the study's source population of asylum seekers in Saxony were available from the State of Saxony [11]. The study was deemed exempt by the University of California Davis Institutional Review Board (2 February 2016, IRB \# 852673-1) and approved by the Kassenärztliche Vereinigung Sachsen Ethics Committee in Dresden. Patient consent was not obtained, as the data were de-identified.

\section{Data analysis}

Only patients with demographic, insurance, and diagnosis data were included in the analyses. Private insurance holders were excluded, as were patients missing insurance data, diagnosis data, or those who were unable to be matched between the insurance and diagnosis databases. The last recorded insurance status was used in the demographic analyses for patients who changed insurance during the study period. Many patients had more than one ICD-10 diagnosis per visit and all the ICD-10 codes were included in analyses. When a new diagnosis was made as part of a patient encounter, providers had the option of specifying whether it was suspected, confirmed, or ruled out. We thus categorized the ICD-10 codes by validity as either suspected, confirmed, or ruled out. Ruled out ICD-10 codes were excluded except for UTI's.

Age and gender characteristics of each diagnosis group were compared, as was the insurance composition of

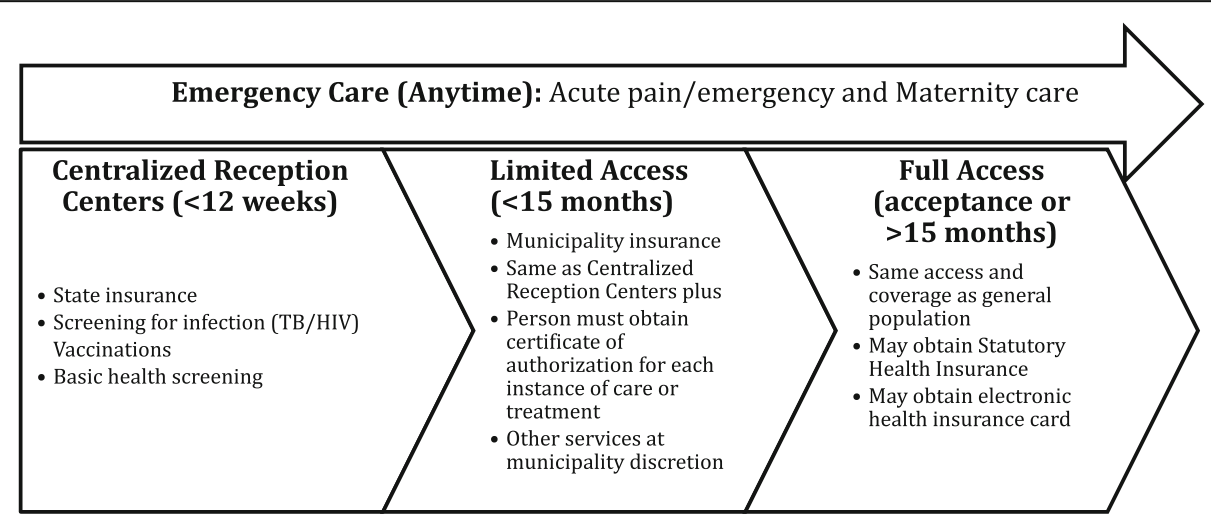

Fig. 1 Germany's asylum seeker healthcare schema. After first arrival, asylum seekers can receive emergency care at any time, and receive screening at centralized reception centers. During this initial period up to 12 weeks, asylum seekers have State insurance. After 3 months, limited access is granted and insurance is provided by the municipality. This lasts up to 15 months, after which full access is possible. Permission to base this figure on a similar one previously published was granted by the author S. Bauhoff [22] 
each diagnosis group, using student's t-test, MannWhitney U test, chi-squared or Fisher's exact tests, as appropriate. The additional category of chronic diseases was created, to include non-communicable diseases and was analyzed as per the other diagnostic groups. Ninetyfive percent confidence intervals were calculated. All analyses were completed using Stata 14 [14].

\section{Results}

After excluding 43 private insurance holders, 2753 nonduplicated patients were seen in the clinic between 14 September 2015 and 31 December 2015. There were 6423 ICD-10 codes. After eliminating missing diagnosis data, insurance data, and non-asylum seeking status, 6361 ICD-10 codes were used for the analysis. There were 4291 unique clinic visits. Ninety ICD-10 codes that were listed but were clinically ruled out were excluded from analyses. However, those patients with ruled out diagnoses were included in the demographic summaries.

\section{Demographics}

Ages ranged from 1 to 87 years, with a mean of 25.3 and standard deviation of 14.9 years, median 25, interquartile range 16-34 (see Additional file 1). Of the 2753 included patients, 1967 (71.5\%) were male, and 786 (28.5\%) were female. There were 1949 patients $(70.8 \%)$ over the age of 18 years and $804(29.2 \%)$ aged 18 or under. Only 29 (1.1\%) were over 65 years of age.

The State Directorate of Saxony's data indicate that 42.5\% were from Syria, 14.6\% from Afghanistan, 11.7\% from Iraq, and the remainder as illustrated in Additional file 2 [11].

Most patients were insured by the state of Saxony (2232 or $81.1 \%$ ), a minority insured by the city of Dresden $(472,17.1 \%)$, and fewer by youth or other asylum-seeker insurance $(49,1.8 \%$, see Table 1$)$. Females comprised 794 or $28.4 \%$ of the overall of the clinic population; a greater number were insured by the state $(n=697$, comprising $31.2 \%$ of those insured by the state) than by the city $(n=75,16.9 \%$ of those insured by the city).
Unaccompanied minors were insured through the Youth Ministry and represented $1.6 \%$ of the clinic population.

Most diagnoses occurred in recently arrived refugee/ state-insured group (82.4\%, $n=5170$, 95\% CI 81.5-83.3). Meanwhile, $16.0 \%(n=1101)$ of diagnoses occurred in those residing in Germany over 3 months and had other insurance.

\section{Diagnostic groups and diagnoses}

There were 6271 diagnoses comprising 683 different diagnostic codes. The most frequent categories were respiratory (J series, $n=1090$ ), miscellaneous symptoms and otherwise not classified ailments ( $\mathrm{R}$ series, $n=881$ ), infection (A and B series, $n=678$ ), musculoskeletal or connective tissue ( $M$ series, $n=583$ ), gastrointestinal ( $\mathrm{K}$ series, $n=427$ ), injury or poisoning ( $\mathrm{S}$ and $\mathrm{T}$ series, $n=372$ ), and mental or behavioral (F series, $n=322$, see Table 2 and Additional file 1: Figure S1).

\section{Respiratory (J00-J98.9): $n=1090$ diagnoses, $n=849$ patients}

Respiratory diagnoses affected over one-third of the clinic population. Of those diagnoses, the most frequent were acute upper respiratory infection $(n=452)$, acute tonsillitis, and bronchitis. Asthma comprised less than $3 \%$ of diagnoses.

Respiratory diagnoses occurred more often in female, in younger and recently arrived patients when compared with the clinic population as a whole. Male patients comprised $67.5 \%$ of those with respiratory diagnoses (95\% CI 64.3-70.6), compared with $71.4 \%$ of the clinic population (95\% CI 69.7-73.1, $p=0.029$, see Table 2.) The mean age was 21.1 (95\% CI 20.0-22.1), significantly lower than the overall clinic population (mean 25.3, 95\% CI 24.8-25.9), and $43.3 \%$ of patients with respiratory diagnoses were minors (95\% CI 40.0-46.7). Of the respiratory patients, 87. $3 \%$ were recently arrived and insured by the state $(95 \% \mathrm{CI}$ $84.9-89.4)$, compared with $81 \%$ of the total clinic population ( $p<0.001$, see Fig. 2 and Additional file 3: Figure S3).

Table 1 Patient demographics and insurance status

\begin{tabular}{llllllll}
\hline & $\begin{array}{l}\text { Total } \\
(n=2753)\end{array}$ & $\begin{array}{l}\text { State Insurance } \\
(n=2232,81.1 \%)\end{array}$ & $\begin{array}{l}\text { Combined Other: } \\
\text { All Non-State } \\
(n=521,18.9 \%)\end{array}$ & $\begin{array}{l}\text { City of Dresden } \\
(n=472,17.1 \%)\end{array}$ & $\begin{array}{l}\text { Unaccomp-anied } \\
\text { Youth }(n=45,1.6 \%)\end{array}$ & $\begin{array}{l}\text { Other } \\
(n=4,0.15 \%)\end{array}$ & $\begin{array}{l}p \text {-value, } \\
\text { State vs. Non-State } \\
\text { Insurance }\end{array}$ \\
\hline $\begin{array}{l}\text { Age in years } \\
\text { (mean (SD) }\end{array}$ & $25.3(14.9)$ & $25.2(15.6)$ & $26.1(11.9)$ & $27.0(12.0)$ & $16.0(2.94)$ & $30.0(10.0)$ & 0.0476 \\
Male (n, \%) & $1967(71.4)$ & $1535(68.8)$ & $432(82.9)$ & $385(81.6)$ & $43(95.6)$ & $4(100)$ & $<0.001$ \\
Minors (n, \%) & $804(29.2)$ & $683(30.6)$ & $121(23.2)$ & $76(16.1)$ & $45(100)$ & 0 & 0.001 \\
Elderly (n, \%) & $29(1.1)$ & $28(1.3)$ & $1(0.2)$ & $1(0.2)$ & 0 & 1 & 0.017 \\
\hline
\end{tabular}

Patient mean age, number and proportion with male gender, number and proportion minors, and number and proportion elderly, in entire population and by insurance status. State insurance is granted upon first arrival in Germany. Other insurance statuses require greater than 3 months in Germany to obtain, indicating these patients were in the country over 3 months. The last known insurance status of patients seen more than once was used. Mann-Whitney $U$ test was used to obtain $p$ values for continuous outcomes, and Pearson's chi-squared test for binary outcomes, comparing state to non-state insurance, 1-sided Fisher's exact chi-squared was used for the elderly category 
Table 2 Patient demographics by diagnosis category

\begin{tabular}{|c|c|c|c|c|c|c|c|c|}
\hline $\begin{array}{l}\text { Diagnosis category } \\
\text { (ICD-10 Group) }\end{array}$ & $\begin{array}{l}\text { Diagnoses } \\
(n, \%)\end{array}$ & Patients (n) & $\begin{array}{l}\text { Age } \\
\text { (mean years } \pm \mathrm{SD} \text { ) }\end{array}$ & $\begin{array}{l}\text { Age } \\
95 \% \mathrm{Cl}\end{array}$ & $\begin{array}{l}\text { Proportion Male } \\
(95 \% \mathrm{Cl})\end{array}$ & $\begin{array}{l}P \text {-Value } \\
\text { Male }\end{array}$ & $\begin{array}{l}\text { Proportion Minors } \\
(95 \% \mathrm{Cl})\end{array}$ & $\begin{array}{l}P \text {-Value } \\
\text { Minors }\end{array}$ \\
\hline Total & $6271(100)$ & 2753 & $25.3 \pm 14.9$ & $24.8-25.9$ & $0.71(0.70-0.73)$ & - & $0.29(0.28-0.31)$ & - \\
\hline Respiratory (J) & $1090(17.4)$ & 849 & $21.1 \pm 15.9$ & $20.0-22.1$ & $0.68(0.64-0.71)$ & 0.029 & $0.43(0.4-0.47)$ & $<0.001$ \\
\hline Miscellaneous Abnormalities (R) & $881(14.1)$ & 667 & $26.1 \pm 14.8$ & $25.0-27.3$ & $0.69(0.66-0.73)$ & 0.215 & $0.26(0.22-0.29)$ & 0.057 \\
\hline Infection (A, B) & $678(10.8)$ & 546 & $18.9 \pm 14.3$ & $17.7-20.1$ & $0.71(0.67-0.75)$ & 0.925 & $0.47(0.43-0.51)$ & $<0.001$ \\
\hline $\begin{array}{l}\text { Musculoskeletal/ } \\
\text { Connective Tissues (M) }\end{array}$ & $583(9.3)$ & 384 & $32.6 \pm 12.9$ & $31.3-33.8$ & $0.73(0.69-0.78)$ & 0.415 & $0.083(0.06-0.12)$ & $<0.001$ \\
\hline Digestive (K) & $427(6.8)$ & 314 & $27.8 \pm 14.6$ & $26.2-29.4$ & $0.75(0.70-0.80)$ & 0.161 & $0.21(0.17-0.26)$ & 0.002 \\
\hline Injury/ Poisoning $(S, T)$ & $372(5.9)$ & 261 & $26.1 \pm 12.4$ & $24.5-27.6$ & $0.84(0.79-0.88)$ & $<0.001$ & $0.23(0.18-0.29)$ & 0.034 \\
\hline Mental/ Behavioral (F) & $322(5.1)$ & 172 & $29.4 \pm 12.2$ & $27.5-31.2$ & $0.65(0.58-0.72)$ & 0.077 & $0.15(0.1-0.21)$ & $<0.001$ \\
\hline
\end{tabular}

The diagnosis categories included patients with ruled out diagnoses in those categories, and the categories are not mutually exclusive. Tests of proportion (chi-squared) were used to obtain $p$-values. Not shown are categories comprising fewer than $5 \%$ of diagnoses

Miscellaneous signs and symptoms (R00-R94.2): $n=881$ diagnoses, $n=667$ patients

The most common symptom was other and unspecified abdominal pain (R10.4, $n=130,14.8 \%$ ), followed by headache (R51, $n=121,13.7 \%)$, cough ( $n=64,7.26 \%)$, pain in throat $(n=44,4.99 \%)$, unspecified pain $(n=44$, $4.99 \%)$, and heartburn $(n=34,3.86 \%)$. The remaining symptoms or signs ranged from dysuria to epistaxis, jaundice, and malaise, to halitosis.

Miscellaneous diagnoses trended mostly male (457, $68.8 \%$ ), not significantly different from the clinic population. The mean age was 25.0, not significantly different from the clinic population. The proportion of minors $(25.5 \%)$ did not reach statistical significance $(p=0.0568)$. The proportion of newly arrived was not statistically different from the proportion of the entire population affected $(p=1.0)$.

Infection (A03.9-B99): $\boldsymbol{n}=\mathbf{6 7 8}$ diagnoses, $\boldsymbol{n}=\mathbf{5 4 6}$ patients Unspecified viral infections were most frequent infectious diagnosis (B34.9, $n=160,23.6 \%$ ), followed by gastroenteritis/colitis (A09.9, $n=114,16.8 \%$ ), scabies (B86, $n=96,14.2 \%$ ), unspecified mycosis (B49, $n=33,4$. $87 \%$ ), and respiratory tuberculosis (A16.9, $n=26,3.83 \%$ ).

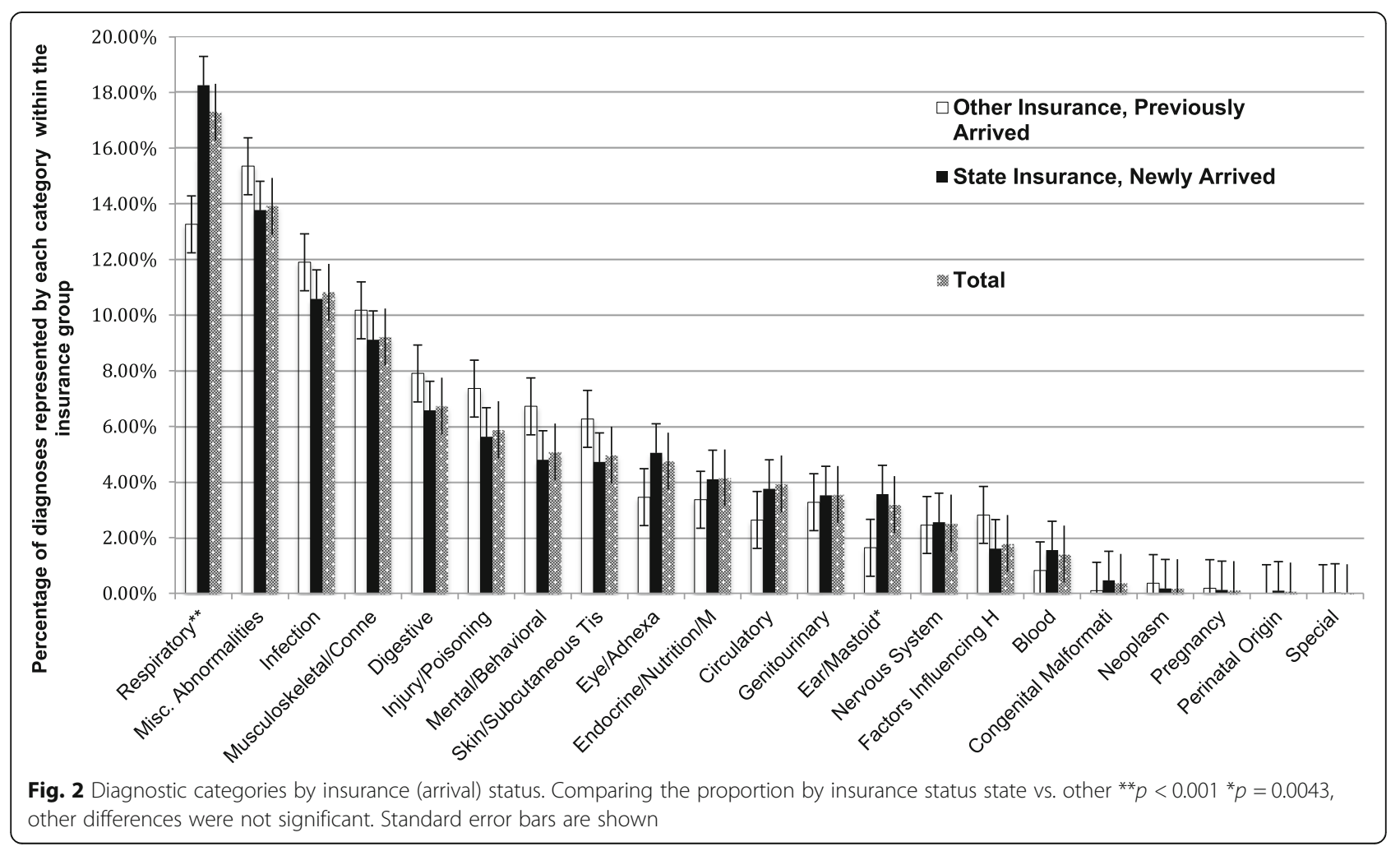


There were two diagnoses of tuberculous pleurisy (A16. 5), one diagnosis of mycoplasma pneumonia (B96.0). There were no HIV diagnoses, six acute hepatitis B infections, five chronic hepatitis B infections, and nine chronic hepatitis $\mathrm{C}$ diagnoses. Six sexually transmitted infections were diagnosed, including gonorrhea, herpes, and chlamydia. Superficial infestations accounted for 204 diagnoses or $30.1 \%$ skin diagnoses, including scabies, superficial mycosis, viral warts, candidiasis of skin and nail, pediculosis, tinea corporis, and tinea pedis. Minors most commonly presented with unspecified viral infections (B34.9, $n=104,31.9 \%$ ), followed by gastroenteritis and colitis (A09.9, $n=77,23.6 \%$ ), and scabies (B86, $n=23,7.06 \%$ ). Of the 18 patients with suspected $\mathrm{TB}$, ten were minors.

Patients with infections trended male (384, 71.2\%), no different from the entire population. Those with infections had a low mean age: 18.9 (95\% CI 17.7-20.1). A significantly higher proportion of those with infections were minors as compared with the entire clinic population (46.9\%, 95 CI 42.7-51.1\%, $p<0.001)$. Insurance type did not correlate with the proportion of those with infectious diseases (see Table 2).

Musculoskeletal (M06.9-M94.0): $n=583$ diagnoses, $n=384$ patients.

The most common musculoskeletal diagnoses were pain in joint (M25.5, $n=112,19.2 \%)$, pain in limb (M79.6, $n=93,16.0 \%$ ), and unspecified dorsalgia (M534.9, $n=92$, $15.8 \%$ ). Less common were low back pain (M54.5, $\mathrm{n}=38$, $6.52 \%$ ), followed by radiculopathy (M54.1, $n=36,6.17 \%$ ), myalgia (M79.1, $n=16,2.74 \%$ ), unspecified arthrosis, and arthritis of rheumatoid and unspecified types. There were nine osteomyelitis diagnoses (M86.9), six unspecified osteonecrosis (M87.9), and four diagnoses of gonarthrosis (M17.9).

Musculoskeletal diagnoses trended male (73.4\%), not different from the population. The mean age was 32.6 (95\% CI 31.3-33.8), significantly older than the whole clinic population. Only $8.30 \%$ of the group with musculoskeletal diagnoses was minor (95\% CI 5.90-11.6\%) less than the total group $(29.2,95 \%$ CI $27.5-30.9 \%, p<0.001)$. Most were recently arrived and insured by the state (78.9\%), not significantly different from the source clinic population.

Digestive or gastrointestinal (K00-K92.9): $n=427$ diagnoses, $n=314$ patients

Gastrointestinal diagnoses included constipation (K59.0, $n=95,22.3 \%$ ), gastritis unspecified (K29.7, $n=65,15.2 \%$ ), and other specified disorders of teeth (K08.8, $n=53$, 12.4\%), followed by hemorrhoids (K64.9, $n=44,10.3 \%$ ), inguinal hernia (K40.9, $n=14,3.28 \%$ ), anal fissure (K60.2, $n=10,2.34 \%)$, gastrointestinal reflux disease (K21.9, $n=9$, 2.11\%), and melena (K92.1, $n=8,1.87 \%$ ).
Gastrointestinal diagnoses were mostly among males (75.2\%), reflecting the population. The mean age of those with gastrointestinal disorders was 27.8 years (95\% CI 26.2-29.4), significantly older than the mean of the clinic population (25.3 years, $95 \%$ CI $24.8-25.9$ ). The proportion of minors with a gastrointestinal diagnosis was significantly lower than published in other studies (20.7, 95\% CI 16.6-25.5\%, $p=0.0015$ ) [9]. The proportion of newly arrived (78.9\%) was not significantly different from the source population.

\section{Injuries and poisoning (S00-T88.7): $n=372$ diagnoses, $n=261$ patients}

The most common injury category was unspecified (T14.9, $n=77,20.7 \%$ ), followed by post-traumatic wound infection (T79.3, $n=24,6.45 \%$ ), and fracture of unspecified body region (T14.2, $n=22,5.91 \%)$. There were 154 fracture diagnoses comprising $41.4 \%$ of the injury diagnoses. Open wounds (T14.0, $n=16,4.3 \%$ ), and superficial injury of unspecified body part (T14.1, $\mathrm{n}=16,4.3 \%$ ) were next most numerous. Sprain and strain of ankle $(n=10,2.69 \%)$ and dislocation, sprain, and strain of other body part $(\mathrm{n}=10,2.69 \%)$ were less common.

Injury diagnoses were more male $(83.9 \%$, 95 CI $78.9-$ $87.9 \%)$ than the general clinic population $(71.4,95 \% \mathrm{CI}$ 69.7-73.1, $p<0.001)$. The mean age was 26.1 (95\% CI 24.5-27.6), while the overall clinic population mean was 25.3. More of the injured patients were minors (32, 95\% CI $18.3-28.5 \%, p=0.034$ ). Similar to the entire clinic population, most injured patients were newly arrived (77.4, 95\% CI 72.3-82.5\%, $p=0.147$ ).

\section{Mental behavioral (F00-F99): $n=322$ diagnoses, $n=172$ patients}

The most common mental health diagnosis was posttraumatic stress disorder (PTSD F43.1, $n=57,17.7 \%$ of mental health diagnoses), followed by unspecified depressive episode (F32.9, $n=41,12.7 \%$ ), adjustment disorders (F43.2, $n=34,10.6 \%)$, somatization disorder (F45.0, $n=22,6.83 \%$ ), and unspecified somatoform disorder (F45.9, $n=18,5.59 \%$ ). The total diagnosis count of PTSD, adjustment, stress reaction, and somatoform disorders (F43.0-F45.9) combined was 153 or $47.5 \%$ of the category. Mental or behavioral disorders related to substance use, dependence, or abuse (F10.1-F19.2), accounted for 18 diagnoses.

A non-significantly lower percentage of patients with mental or behavioral diagnoses were male in comparison to the rest of the clinic population $(65.1,95 \%$ CI $57.8-71$. $8 \%, p=0.0772$ ). The mean age of 29.4 (95\% CI 11.8-21.9) is not significantly different from the clinic population. However, only $14.5 \%$ of patients with these diagnoses were minors (95\% CI 10.0-20.6), compared with $29.2 \%$ overall $(p<0.001)$. Most of the patients were newly arrived with 
no difference from the clinic population $(80.2,95 \%$ CI 73 . $6-85.5 \%, p=0.77)$.

\section{Skin or subcutaneous (L00-L99): $n=313$ diagnoses, $n=\mathbf{2 4 9}$ patients}

The most common skin diagnoses were unspecified dermatitis (L30.9, $n=59,18.9 \%$ ), abscess, furuncle or carbuncle (L02.9, $n=30,9.58 \%$ ), unspecified acne (L70.9, $n=30,9.58 \%$ ), psoriasis (L40.9, $n=26,8.31 \%$ ), unspecified pruritis (L29.9, $n=22,7.03 \%$ ), local infection (L08.9, $n=15,4.79 \%$ ), and unspecified follicular disorder (L73.9, $n=14,4.47 \%)$.

Similarly to the general clinic population, patients with skin or subcutaneous diagnoses trended male (75.9\%, 95 CI $70.2-80.8 \%, p=0.131)$, but had a lower mean age at 22.3 years (95\% CI 20.6-24.0). Minors comprised 36.5\% (95\% CI 30.8-42.7\%) of the patients compared with $29.2 \%$ overall $(p=0.0159)$. There was no significant difference in the proportion of newly arrived compared with the clinic population (79.9, 95\% CI 74.5-84.4\%).

\section{Genitourinary (N00-N099): $n=218$ diagnoses, $n=212$ patients}

Urinary tract infection (UTI) was the most common genitourinary diagnosis, occurring 42 times (19.3\%), followed by cystitis, unspecified (N30.9, $n=22,10.1 \%)$, acute vaginitis $(N=76.0, \mathrm{n}=21,9.63 \%)$, and calculus of kidney (N20.0, $n=18,8.26 \%$ ). UTI was ruled out in 35 cases.

Genitourinary diagnoses were mostly diagnosed in female patients; only $43.4 \%$ were in males (95\% CI $36.9-$ $50.1 \%, p<0.001)$. The mean age was not significantly different from the clinic population at 26.2 (95\% CI 24. 3-28.2). Minors made up $25.0 \%$ of this group, not significantly different from the general clinic population. Most (83\%) were newly arrived and insured by the state.

\section{Pregnancy-related (O00-099, Z33, Z34): $n=49$ diagnoses,} $n=\mathbf{4 0}$ patients

Of the 786 female patients, there were 40 non-duplicated patients pregnant at the time of data collection $(5.09 \%$ of female patients). The mean age was 26.0 years (95\% CI 24.1-27.8), and five were minors (12.0\%, 95 CI 2.30$22.7 \%, p=0.0208)$. Most (72.5\%) were recently arrived and insured by the state, not significantly different from the general clinic population.

Chronic diseases (see Additional file 4: Table S1 for ICD10 codes included): $n=\mathbf{5 1 9}$ diagnoses, $n=\mathbf{2 9 3}$ patients

Chronic conditions excluding mental health diagnoses were present in 293 patients. The most common diagnoses were hypertension (I10.9, $n=124)$, unspecified diabetes mellitus (E14.9, $n=77$ ), unspecified epilepsy (G40.0, $n=54)$, and type 2 diabetes mellitus without complication $(\mathrm{E}=11.9, \quad n=53)$. Other common diagnoses included chronic obstructive pulmonary disease $(n=31)$, thalassemia $(n=30)$, and asthma $(n=28)$.

The mean age was significantly higher than the source population (33.6 years, 95\% CI 31.6-35.5). There was a significantly lower proportion of minors (17.1, 95\% CI $13.2-21.8 \%, p<0.001)$. This group was $66.2 \%$ (95\% CI $60.1-71.4 \%)$ male, not significantly different from the clinic population $(p=0.0625)$. There was a nonsignificantly higher proportion of newly arrived patients in this group (85.0, 95\% CI 80.4-88.6\%, $p=0.102)$.

\section{Discussion \\ Epidemiological findings Representativeness of population}

The age and sex of our population were consistent with data on newly arriving refugees from the state of Saxony (Additional file 5: Figure S4) [11]. Because our database did not capture nationality or country of origin, we can only surmise that the patients were similar to the asylum seekers in the state of Saxony at the time, with the majority from Syria, followed by Afghanistan and Iraq [11]. The most requested languages for assistance in the clinic were Arabic, Farsi, and Dari. Eighty percent of patients lived in centers located in and around the city of Dresden, and because our clinic was the only point of first care for refugees in the area, we postulate that most of the ambulatory non-emergency medical care they received occurred in our clinic.

\section{Disease burden profile}

In spite of the common perception of an association between migration and the importation of infectious diseases we did not find such an association in our study as seen in the low number of transmissible infections.

Asylum seekers within 3 months of arrival in Germany, as identified by insurance status, were significantly more likely to have a respiratory diagnosis, mostly infectious, when compared with asylum seekers with three or more months of residence $(p<0.001)$. The preponderance of respiratory infections is consistent with recent studies in Belgium, Germany, and Switzerland [8, $15,16]$. More frequent respiratory diagnoses among new arrivals are likely attributable to conditions in Dresden's reception centers, which often had open sleeping arrangements and minimal temperature control. Exposure and stress during the flight to Germany may also have contributed. In our study, ear and mastoid diagnosis numbers may also be related to flight circumstances. The proportion of minors with a gastrointestinal diagnosis $(20.7 \%)$ was significantly lower than that published in other studies (50\%) [9].

Non-Communicable Diseases (NCD) represent a newly recognized challenge in refugee operations [17]. However, this population had fewer NCD diagnoses (10.8\%), as 
compared with the age-matched (19-29 years) population 16.3\% (95\% CI 14.4-18.4\%) and the general population 38. 8\% (95\% CI 37.9-39.7\%) in Germany [18]. The lower level of NCDs documented in our clinic may either be due to self-selection at the time of immigration, decreased careseeking among patients with chronic diseases, or even missed diagnoses. There may also be a change in the composition of the patient population over time, as the initial migrations during the summer of 2015 were primarily by foot, whereas in the fall and winter months, more asylum seekers travelled by bus and train [19]. Except for certain documented cases of asylum seekers with mobility problems traveling the Balkan corridor during the summer 2015, most people seeking refuge had to be physically fit and healthy enough to endure the difficult conditions associated with the journey. This changed in late Fall and early Winter 2015, when buses or trains were made available to shuttle people across countries along the Balkan route [20]. This possibly enabled a higher proportion of people with physical disabilities or physically limiting NCDs to undertake the journey. Our study sample did not demonstrate higher rates of NCDs among longer-established refugees compared with newly arrived refugees.

One diagnostic category inconsistent with prior literature is the higher level of eye diagnoses [21]. Soon after the clinic opened, a major optical firm donated 160 vouchers for new glasses for qualified patients and may account for the overrepresentation of eye-related diagnoses in our study.

The mental health prevalence documented in refugee populations in Germany is variable, ranging from 6.65$76.66 \%$ for institution-based studies such as ours, to 16 . $36-54.90 \%$ in population based studies [4]. In comparison to these studies, the mental health prevalence in our sample appears low. This underrepresentation of mental health prevalence in our sample may be explained because 1) mental health disorders often presented as non-specific pain or psychosomatic complaints to the primary care physicians and 2) the psychiatrists, who carried out the majority of mental health diagnoses in our clinic, had limited patient appointment availability. The reliability and validity of the mental health diagnoses in our study are thus difficult to ascertain.

\section{Limitations}

The short time frame of available data limits this study. Another limitation is the absence of patient demographic data. Data not directly linked to medical care could not be collected due to German law. Another limitation is the use of ICD-10 diagnostic categories as a proxy of disease prevalence: this prevents weighing diagnoses by order of importance in patients receiving multiple diagnoses, which leads to a low-resolution epidemiological topography.

\section{Conclusions}

This descriptive epidemiological study confirms previous findings of disease prevalence in newly arrived asylum seekers. Common health problems comprise communicable diseases including upper respiratory, gastrointestinal and skin infections, injuries and wounds, as well as the psychiatric sequelae of trauma. Aside from upper respiratory infections, no significant differences in disease profile between newly arrived and longer settled refugees were found.

\section{Keypoints}

- Our findings are similar to previously published studies from Germany and Belgium documenting how the first migratory wave consists of mostly male, relatively young and relatively healthy individuals.

- Asylum seekers in Dresden did not bear a high burden of communicable diseases, but several warranted additional screening and treatment, including for tuberculosis and scabies.

- Respiratory illnesses were more common amongst those who had been in Germany less than 3 months compared with those residing longer, and may indicate a need for improved care and conditions immediately upon arrival and during flight.

- Nearly half of the mental health disorders were trauma-related (PTSD, adjustment, stress reaction, and somatoform disorders).

- The prevalence of chronic disease in this study was relatively low.

- This study helps determine which primary care based screening and treatment interventions may be most appropriate for a population of asylum seekers in Europe.

\section{Additional files}

Additional file 1: Figure S1. Patient age and gender distribution. All asylum seeker patients seen in the period 14 September to 31 December 2015 are plotted by age and gender, including those with ruled-out diagnoses, by five-year increments. (DOCX 64 kb)

Additional file 2: Figure S2. Distribution of ICD-10 diagnosis categories. Number of diagnoses are shown on the Y-axis. (DOCX $60 \mathrm{~kb}$ )

Additional file 3: Figure S3. Fifteen most common diagnoses. The fifteen most common diagnoses by ICD-10 codes are plotted with the number of diagnoses for each code on the Y-axis. (DOCX $71 \mathrm{~kb}$ )

Additional file 4: Table S1. Diagnoses included in chronic conditions. (DOCX $95 \mathrm{~kb}$ )

Additional file 5: Figure S4. Countries of origin of asylum seekers in Saxony 2015. Countries of origin are plotted with percentage of total asylum seekers who arrived in Saxony between 1 January and 31 December 2015. The data is from Landesdirektion Sachsen, or the State Directorate of Saxony [11]. (DOCX $77 \mathrm{~kb}$ ) 


\section{Abbreviations}

Cl: Confidence interval; ICD-10: International Statistical Classification of Diseases and Related Health Problems, 10th revision; KVS: Kassenärztliche Vereinigung Sachsen (regulating body for outpatient care in Saxony); LDS: Landesdirektion Sachsen (Government of Saxony); NCD: Non-communicable diseases; SD: Standard deviation; TB: Tuberculosis; UTI: Urinary tract infection; WHO: World Health Organization

\section{Acknowledgements}

Thank you Brian Healy, KVS, Mr. Rabe, and Marc Parenteau for your support.

\section{Availability of data and materials}

The data that support the findings of this study are available from KVS but restrictions apply to the availability of these data, which were used under license for the current study, and so are not publicly available. Data are however available from the authors upon reasonable request and with permission of KVS.

\section{Authors' contribution}

LG: conceptualization, data collection, data cleaning, and analysis, writing manuscript. GJ: critical revisions of manuscript. JG: conceptualization and critical revisions of manuscript. DF: critical revisions of manuscript. ST: conceptualization, guiding of analysis, writing manuscript. All authors have read and approved of the final version of the manuscript.

\section{Ethics approval and consent to participate}

This research using human data was conducted in accordance with the Declaration of Helsinki. The study proposal was reviewed by the University of California Davis Institutional Review Board on Feb 2, 2016 (IRB \# 852673-1) and designated as exempt. KVS approved the study and provided the data. Written informed patient/subject consent was not obtained for this study, which used de-identified data in a retrospective fashion.

\section{Competing interests}

The authors declare that they have no competing interests.

\section{Publisher's Note}

Springer Nature remains neutral with regard to jurisdictional claims in published maps and institutional affiliations.

\section{Author details}

'Department of Surgery, University of California Davis Health, 2315 Stockton Blvd, OP 512, Sacramento, CA 95817, USA. ²General Medicine Division, MK3, Technical University Dresden, Carus School of Medicine, Fetscherstrasse 74, 01307 Dresden, Germany.

Received: 15 January 2018 Accepted: 2 May 2018

Published online: 16 May 2018

\section{References}

1. The United Nations High Commissioner for Refugees. Convention and Protocol Relating to the Status of Refugees. http://www.unhcr.org/protec tion/basic/3b66c2aa10/convention-protocol-relating-status-refugees.html. Accessed 12 Apr 2016

2. Eurostat Statistics Explained: Asylum Statistics. http://ec.europa.eu/eurostat/ statistics-explained/index.php/Asylum_statistics\#Asylum_applicants. Accessed 5 Dec 2016.

3. Migration, asylum, and refugees in Germany: Understanding the data. Berlin, Germany: Global Migration Data Analysis Centre (GMDAC) of the International Organization for Migration (IOM), 2016. https://www.iom.int/ news/migration-asylum-and-refugees-germany-understanding-data. Accessed 13 Apr 2016

4. Bozorgmehr K, Mohsenpour A, Saure D, et al. Systematische Übersicht und „Mapping" empirischer Studien des Gesundheitszustands und der medizinischen Versorgung von Flüchtlingen und Asylsuchenden in Deutschland (1990-2014) (Systematic Review and Mapping of Empirical Studies on Health and Health Care of Asylum-Seekers and Refugees in Germany (1990-2014)). Bundesgesundheitsblatt 2016;59(5): 599-620.

5. van Berlaer $G$, Bohle Carbonell F, Manantsoa S, et al. A refugee camp in the Centre of Europe: clinical characteristics of asylum seekers arriving in Brussels. BMJ Open. 2016;6(11):e013963.
6. Mohammadzadeh $Z$, Jung F, Lelgemann M. Health for refugees - the Bremen model. Bundesgesundheitsblatt. 2016:59(5):561-9.

7. Amara AH, Aljunid SM. Noncommunicable diseases among urban refugees and asylum-seekers in developing countries: a neglected health care need. Glob Health. 2014;10:24

8. Khan MS, Osei-Kofi A, Omar A, et al. Pathogens, prejudice, and politics: the role of the global health community in the European refugee crisis. Lancet Infect Dis. 2016;16(8):e173-7.

9. Marquardt L, Kramer A, Fischer F, et al. Health status and disease burden of unaccompanied asylum-seeking adolescents in Bielefeld, Germany: crosssectional pilot study. Tropical Med Int Health. 2015; https://doi.org/10.1111/ tmi.12649.

10. Bundesministerium der Justiz und für Verbraucher Schutz (German Federal Ministry of Justice and Consumer Protection). Asylbewerberleistungsgesetz (AsylbLG) § 4 Leistungen bei Krankheit, Schwangerschaft und Geburt. (Asylum seeker laws governing health, pregnancy, and birth). https://www. gesetze-im-internet.de/asylblg/ 4.html. Accessed 5 Dec 2016.

11. Landesdirektion Sachsen (Government of Saxony). FAQ zum Asyl (Asylum FAQ). https://www.lds.sachsen.de/asyl/?ID=9260\&art_param=720. Accessed 13 Apr 2016.

12. Deutsche Presse-Agentur $\mathrm{GmBH}$. Flüchtlingsambulanz in Dresden: Dienst fern des Normalen (Refugee clinic Dresden: service far beyond normal). Dresdener Neueste Nachtrichten (Dresden's Newest Reports). http://www. dnn.de/Dresden/Lokales/Fluechtlingsambulanz-in-Dresden-Dienst-fern-desNormalen. Accessed 7 Jan 2016.

13. Evangelische Hochschule Dresden, Forschung Zentrum (Evangelical University of Dresden Research Center). Wissenschaftliche Begleitung des Projekts „Praxis zur ambulanten Versorgung von Asylbewerberinnen und Asylbewerbern" (Scientific Summary of the Project: Medical Gatekeepers for Asylum Seekers in Dresden). http://www.ehs-dresden.de/index.php?id=722. Accessed 7 Feb 2016

14. StataCorp. Stata statistical software: release 14. College Station: StataCorp LP. 2015.

15. Economopoulou A. Spectrum of diseases occurring in refugees and asylum seekers: data from three different medical institutions in the Munich area from 2014 and 2015. Dtsch Med Wochenschr. 2016 Jan;141(1):e8-15.

16. Pfortmueller CA, Schwetlick M, Mueller T, et al. Adult asylum seekers from the Middle East including Syria in Central Europe: what are their health care problems? PLoS One. 2016;11(2):e0148196.

17. Doocy S, Lyles E, Roberton T, et al. Prevalence and care-seeking for chronic diseases among Syrian refugees in Jordan. BMC public health. 2015;15:1097. Robert Koch-Institut (Hrsg) (2012) Daten und Fakten: Ergebnisse der Studie "Gesundheit in Deutschland aktuell 2010." (Data and facts: Findings of the study "Health in Germany update 2010). Beiträge zur Gesundheitsberichterstattung des Bundes (Federal Health Reports). RKI, Berlin.

18. Robert Koch-Institut (Hrsg). Daten und Fakten: Ergebnisse der Studie "Gesundheit in Deutschland aktuell 2010." Beiträge zur Gesundheitsberichterstattung des Bundes, 2012. RKI, Berlin.

19. European Commission, Strasbourg. Final report from the Commission to the European Parliament and the Council on the follow up to the Leader's Meeting on the refugee flows along the Western Balkans Route. Com (2015) 676. https://ec.europa.eu/home-affairs/sites/homeaffairs/files/what-we-do/ policies/securing-eu-borders/legal-documents/docs/report_western_balkans _en.pdf, Accessed 1 Nov 2017.

20. Editors, Border Monitoring.Eu. Analysis "The Summer of Migration Part 6 the 'Humanitarian Corridor.' 11 Sept 2016. http://moving-europe.org/thehumanitarian-corridor/\# ftn1. Accessed 15 Apr 2017.

21. Alberer $M$, Wendeborn $M$, Loescher $T$, et al. Erkrankungen bei Fluechtlingen und Asylbewerbern (IIlnesses of refugees and asylum seekers). Dtsch Med Wochenschr. 2016;141:e8-e15.

22. Göpffarth, D, Bauhoff, S. The Public Health Dimension of Germany's Refugee Crisis. http://healthaffairs.org/blog/2015/10/22/the-public-health-dimensionof-germanys-refugee-crisis/. Accessed 5 Dec 2016. 